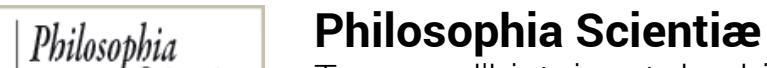

Scientie Travaux d'histoire et de philosophie des sciences

14-1 | 2010

Varia

\title{
Sur le voir humain (1855)
}

\section{Hermann von Helmholtz}

Traducteur : Christophe Bouriau

\section{CpenEdition}

Journals

Édition électronique

URL : http://journals.openedition.org/philosophiascientiae/152

DOI : 10.4000/philosophiascientiae.152

ISSN : $1775-4283$

Éditeur

Éditions Kimé

Édition imprimée

Date de publication : 1 avril 2010

Pagination : 13-35

ISBN : 978-2-84174-521-0

ISSN : 1281-2463

\section{Référence électronique}

Hermann von Helmholtz, « Sur le voir humain (1855) », Philosophia Scientiæ [En ligne], 14-1 | 2010, mis en ligne le 01 avril 2013, consulté le 16 janvier 2021. URL : http://journals.openedition.org/ philosophiascientiae/152 ; DOI : https://doi.org/10.4000/philosophiascientiae.152 


\title{
Sur le voir humain (1855)
}

\author{
Hermann von Helmholtz \\ Traduction de Christophe Bouriau, \\ revue par Alexandre Métraux
}

Assemblée très honorée,

Ce qui nous réunit ce jour, c'est la mémoire d'un homme qui plus qu'aucun autre a contribué à lier indissolublement le nom de notre ville à l'histoire culturelle de l'humanité, je veux parler de Kant, le Philosophe. Nous souhaitons ériger un monument à sa mémoire, pour montrer que notre époque et notre ville nourrissent un souvenir reconnaissant et respectueux envers les hommes qui leur apportent progrès scientifique et instruction. Par la présente conférence, je souhaite contribuer à ma manière à cette marque d'estime et d'admiration. «Quoi? Un savant qui rend hommage à un philosophe? », se diront peut-être certains d'entre vous, passablement informés des récentes orientations scientifiques. N'est-il pas de notoriété publique que le physicien et le philosophe, de nos jours, ne s'entendent pas trop bien, du moins dans leurs travaux scientifiques? Ne sait-on pas qu'un âpre combat les oppose depuis longtemps, et que, s'il semble avoir cessé à l'heure actuelle, cela ne vient certainement pas de ce qu'un parti a convaincu l'autre, mais de ce que chaque parti désespère de convaincre l'autre. On entend les savants se glorifier haut et fort de ce que les grands progrès récemment accomplis dans leurs disciplines respectives sont apparus dès qu'ils ont parfaitement purifié leur domaine des influences de la philosophie de la nature. Ceux d'entre vous qui connaissent cette situation penseront peut-être que ce n'est pas un intérêt sincère pour la chose même qui m'amène ici aujourd'hui, mais des considérations extérieures concernant la ville et l'université que Kant fréquentait jadis et que j'ai l'honneur de fréquenter à mon tour aujourd'hui, à moins que les deux partis en conflit se soient par sagesse entendus pour se présenter sous un jour favorable, en vénérant et honorant des adversaires devenus inoffensifs?

Je vous assure cependant que ce ne sont pas de simples considérations extérieures ou encore une adversité masquée qui me guident, mais une reconnaissance et une vénération véritables. Du reste, le sujet que j'ai choisi pour ma conférence est censé illustrer à quel point les pensées du grand philosophe continuent de vivre et de fructifier aujourd'hui dans des domaines de la science où on s'en serait le moins douté. Le hiatus radical qui sépare la philosophie 
d'aujourd'hui des sciences de la nature n'existait pas à l'époque de Kant. Lorsqu'il pratiquait les sciences de la nature, il s'appuyait sur les mêmes fondements que les physiciens; il s'intéressait vivement, lui aussi, à la théorie newtonienne des mouvements des corps célestes, à cet édifice intellectuel le plus grandiose jamais élaboré par l'entendement humain, au sein duquel l'infinie variété des mouvements célestes se déploie selon la plus rigoureuse nécessité à partir du principe le plus simple, la gravitation universelle. Cet édifice pouvait en même temps être considéré comme le modèle de toutes les théories physiques à venir. De fait, Kant lui-même tenta, en suivant Newton, d'élaborer une hypothèse concernant la genèse de notre système planétaire par la seule force de gravitation. Cette tentative pourrait même nous autoriser à compter le Philosophe parmi les savants.

Les sciences de la nature reposent toujours sur les mêmes principes qu'à l'époque de Kant, ceux dont Newton a exemplairement montré la fécondité; elles n'ont fait que gagner en richesse et leurs principes se sont imposés à un nombre toujours croissant de faits particuliers. Toutefois, la position de la philosophie à leur égard a changé. La philosophie de Kant, pour sa part, ne cherchait pas à augmenter le nombre de nos connaissances au moyen de la pensée pure, son premier principe étant que toute connaissance de la réalité doit être tirée de l'expérience. Elle visait seulement à examiner les sources de notre savoir et son degré de légitimité, entreprise qui incombera toujours à la philosophie et qu'aucun siècle ne pourra lui retirer impunément.

Un autre puissant penseur a débuté son parcours scientifique dans notre ville, sous les auspices de Kant, je veux parler de Fichte. Quelque rude et importante que soit son opposition à la conception ordinaire du monde, autant que je puisse en juger, ses principes n'entrent nullement en conflit avec les sciences de la nature. Au contraire, l'analyse qu'il présente de la perception sensible s'accorde parfaitement avec les conclusions que la physiologie sensorielle a tirées par la suite des faits d'expérience. C'est une partie de ces faits que je souhaite vous exposer aujourd'hui.

En revanche, lorsque, après la mort de Fichte, Schelling s'est mis à dominer la science de l'Allemagne méridionale, et Hegel celle du Nord, un vent de discorde a commencé à souffler. Désormais mécontente du statut que Kant lui avait assigné, la philosophie crut qu'elle avait trouvé de nouvelles voies permettant d'atteindre d'emblée et sans l'expérience, au moyen de la pensée pure, les résultats que les sciences empiriques étaient censées obtenir tôt ou tard. Elle n'hésitait pas à s'approprier toutes les questions ultimes touchant le ciel et la terre, le présent et l'avenir. L'opposition de ces écoles aux principes scientifiques de l'étude de la nature s'exprima de manière particulièrement nette dans la polémique passionnée, a-philosophique au plus haut point, qui opposa Hegel et quelques-uns de ses disciples à Newton et à ses théories. Les sciences de la nature, alors peu fréquentées par les personnes de culture dans une Allemagne qui se passionnait pour la philosophie, eurent le plus souvent le dessous. Qui ne serait tenté de préférer la voie rapide et créatrice de la pensée pure au travail pénible du journalier qui progresse dans l'étude de la 
nature? Quelques rares exceptions honorables parmi les savants allemands, Humboldt, Erman, Pfaff combattirent obstinément, mais dans l'isolement, ce qu'on nommait alors philosophie de la nature, jusqu'à ce que le grand essor des sciences de la nature dans les pays d'Europe voisins n'entraîne également l'Allemagne.

La philosophie avait voulu tout prendre à sa charge. À présent, c'est à peine si l'on était prêt à lui confier ce qui pourrait lui revenir de droit. Mais faut-il s'en étonner, dans une époque où les espoirs les plus audacieux avaient laissé place à un profond découragement, et où les plus récents systèmes de la philosophie, confondus avec la philosophie elle-même, avaient suscité une méfiance qui avait gagné la science toute entière?

Le point sur lequel philosophie et sciences se touchent au plus près est la théorie des perceptions sensibles de l'homme. C'est pourquoi je souhaite vous exposer les résultats scientifiques relatifs à l'organe sensoriel dont les fonctions ont été examinées le plus complètement jusqu'à ce jour, je veux parler de l'œil. Vous pourrez ensuite juger par vous-mêmes du rapport entre les résultats de l'expérience et ceux de la philosophie.

L'œil est un instrument d'optique formé par la nature, une camera obscura naturelle. Je suppose que la majeure partie de l'assistance a déjà assisté à la préparation de daguerréotypes ou d'images photographiques, et qu'elle a un peu observé l'instrument utilisé à cette fin. Cet instrument est une camera obscura, à la construction extraordinairement simple. Il s'agit pour l'essentiel d'une simple caisse de bois noircie à l'intérieur, à une extrémité de laquelle on a disposé une lentille de verre, et à l'autre extrémité une tablette de verre dépoli. Quand le côté de la boîte comportant la lentille est dirigé vers un quelconque objet bien éclairé et que l'instrument est bien réglé, on voit sur la tablette de verre mat une image d'autant plus réduite que l'objet est lointain, image très nettement dessinée et naturellement colorée. Il s'agit toutefois d'une image inversée de l'objet. Après avoir donné à son instrument la position adéquate, le photographe remplace la tablette de verre par une petite plaque d'argent préparée par ses soins, de sorte que l'image qui se projetait sur la plaquette de verre se projette à présent sur le support argenté. L'image reste visiblement conservée sur la petite plaque argentée, dont la surface est spécifiquement modifiée par l'impression de la lumière dans la zone la plus nette de l'image. Les photographies connues de tous ne sont donc en fait que des images fixées dans une camera obscura.

L'œil est un instrument du même genre; la seule différence essentielle avec l'appareil photographique étant qu'au lieu d'une tablette de verre mat ou d'une petite plaque photosensible, on trouve à l'arrière plan de l'œil une peau nerveuse sensible qu'on nomme la rétine, où la lumière provoque des réactions qui sont ensuite transférées au cerveau par les fibres nerveuses rétiniennes réunies dans le nerf optique. Extérieurement, certes, la camera obscura naturelle de l'œil diffère bel et bien de l'artificielle. Au lieu d'une caisse en bois quadrangulaire, vous trouvez le globe oculaire rond, dont la paroi ferme est formée 
par une peau fibrillaire de couleur blanche. On peut observer sur tout homme vivant la partie extérieure de cette paroi, qu'on nomme le blanc de l'œil. La couleur noire qui recouvre l'intérieur du caisson de la camera obscura trouve son équivalent dans l'œil : la choroïde, cette seconde peau plus fine de couleur brune. Sur un œil vivant, nous n'en voyons que l'extrémité saillante, c'est-àdire le cercle bleu ou brun qu'on nomme l'iris. L'iris entoure l'ouverture noire du milieu de l'œil qu'on nomme la pupille. Dans sa partie reculée, l'iris est très noir, comme le reste de la choroïde, tandis que sur sa partie avancée et visible, on trouve des couches dépourvues de couleur ou peu colorées. La couleur bleue de l'iris ne vient pas d'un pigment spécifique; elle s'explique de la même manière que la couleur bleue du lait dilué. Cette couleur est commune aux milieux troubles blancs placés devant un fond sombre. La couleur brune de l'iris provient de la sédimentation, sur ses couches de devant, de petites quantités du pigment brun noir qui recouvre sa surface arrière. Cette coloration peut se modifier si au cours du temps quelque pigment se sédimente dans l'iris. Aristote rapporte déjà que tous les enfants naissent avec les yeux bleus, même si plus tard certains virent au brun. Vous voyez ainsi que les philosophes de l'Antiquité savaient eux aussi observer.

Le cercle noir au milieu du cercle brun ou bleu, qu'on nomme la pupille, est donc une ouverture par laquelle la lumière pénètre dans la partie arrière de l'œil. Si la quantité de lumière est trop grande, la pupille se rétrécit, si elle est très faible, elle s'élargit. Devant la pupille se trouve la cornée transparente, qui a la forme bombée d'un verre de montre, et dont la surface supérieure reste polie comme un miroir grâce à l'humidité lacrymale humectante et grâce au battement des paupières. Derrière la pupille se trouve un autre corps transparent en forme de lentille, le cristallin, dont seuls de faibles reflets lumineux dans l'œil vivant trahissent la présence. L'intérieur de l'œil est d'ailleurs rempli de liquide. À eux deux, le cristallin et la surface courbe de la cornée fonctionnent dans l'œil comme la lentille de verre de la camera obscura du photographe. Ils projettent sur la surface rétinienne placée à l'arrière-plan de l'œil, juste devant la choroïde, des images réduites, aux couleurs naturelles, des objets externes. Il s'agit toutefois d'images inversées. Pour l'observation de la rétine de l'œil vivant, j'ai construit il y a quelques années un petit instrument optique, le miroir oculaire, grâce auquel on peut voir également, de manière directe, les images rétiniennes dans l'œil d'autrui, et s'assurer de leur intensité, de leur position, etc.

On a dit précédemment que le photographe devait ajuster son instrument par rapport à l'objet qu'il voulait prendre. Un examen plus poussé des images dans la camera obscura nous apprend que lorsque les objets éloignés sont pris avec des contours nets, les objets plus proches paraissent informes, et vice versa. Lorsqu'il veut prendre des objets éloignés, le photographe doit rapprocher la lentille de son appareil de la tablette sur laquelle l'image est projetée, et il doit l'en éloigner s'il veut prendre des objets proches. Quelque chose de similaire se produit dans l'œil. Vous ne pouvez pas voir nettement tout à la fois des objets proches et lointains. La meilleure et plus facile manière de vous 
en convaincre est de tenir un voile à six pouces de vos yeux en gardant un œil fermé. Vous pouvez alors regarder à travers le voile, sans modifier l'orientation du regard, tantôt des objets éloignés; le voile vous apparaît alors comme une opacification informe du champ visuel, et vous ne pouvez pas en discerner les divers fils. Et tantôt, inversement, si vous considérez les fils du voile, vous ne pouvez plus identifier nettement les objets à l'arrière-plan. En effectuant cette expérience, on ressent un certain effort dans l'œil lorsque l'on passe d'un point de vue à l'autre. De fait, à cette occasion, la forme du cristallin est délibérément modifiée au moyen des appareils musculaires situés dans l'œil. Cette modification, qui confère à l'œil le pouvoir de se diriger tantôt vers des objets proches, tantôt vers des objets éloignés, est nommée accommodation au proche et au lointain. Grâce au miroir oculaire, on peut aussi observer de manière directe les modifications d'images dues aux modifications de l'accommodation.

Je m'attarde encore un instant sur la nature de l'image optique dans l'œil et sur la nature des images optiques en général. Les rayons qui passent d'un milieu transparent à un autre, par exemple de l'air au verre, ou de l'air au liquide de l'œil, sont déviés de leur direction antérieure; ils sont «brisés » lorsqu'ils ne frappent pas la surface de séparation à angle droit. La lentille de verre de la camera obscura, à l'instar des milieux transparents de l'œil, modifie la trajectoire des rayons lumineux qui viennent d'un point éclairé d'un objet dont nous avons l'image, mais de telle manière que les rayons convergent tous à nouveau en un point, qui est le point correspondant de l'image optique. Lorsque ce point de convergence des rayons lumineux se situe sur la surface de la rétine, il est touché par la totalité de la lumière qui transite du point correspondant de l'objet jusqu'à notre œil, et aucune fraction de cette lumière n'atteint les autres parties de la rétine. Ce point de la rétine n'est donc pas touché par la lumière provenant de n'importe quel autre point de l'objet. Il reçoit toute la lumière et rien que la lumière ayant transité du point correspondant de l'objet dont nous avons l'image jusqu'à notre œil. Si le point émet de la lumière rouge, l'autre point sera illuminé en rouge, et s'il émet de la lumière verte, l'illumination se fera en vert, etc. À chaque point du monde extérieur correspond ainsi un point particulier dans l'image que nous en prenons, point qui possède une intensité lumineuse correspondante et une couleur identique à celle du point original. Ainsi, lorsque l'œil jouit d'une vision claire, chaque point particulier de la rétine correspond à un point particulier du champ visuel externe, de sorte que l'œil n'est frappé et stimulé à ressentir que par la lumière provenant de ce point externe. Puisque, ainsi, chaque point particulier du champ visuel n'affecte par sa lumière qu'un point particulier de la substance nerveuse sensorielle, on peut prendre conscience de la grandeur et de la couleur lumineuse de chaque point externe pris isolément. Cette organisation de l'œil en tant que appareil optique permet de percevoir séparément les différents objets lumineux de notre entourage. Mieux l'élément optique de l'œil remplit sa fonction, plus le discernement des détails du champ visuel est prompt. 
Même s'il présente divers faits et questions dignes d'intérêt, je ne souhaite pas développer cet aspect physique des processus visuels, qu'il me fallait aborder uniquement pour faire comprendre ce qui va suivre. Un seul fait toutefois me servira à illustrer dans quelle mesure notre image du monde externe dépend également de la constitution de la partie physique de notre œil. Les étoiles nous paraissent rayonnantes, et, dans notre langue, « étoilé »a la même signification que «rayonnant». En vérité, les étoiles sont de forme ronde et très souvent si petites que nous ne pouvons en connaître la forme; elles devraient nous apparaître comme des points indivisibles. L'image de l'étoile ne reçoit les rayons ni dans l'espace cosmique ni dans notre atmosphère. C'est notre cristallin, dont la structure est rayonnante, qui lui offre cette parure; les rayons que nous attribuons aux étoiles sont en réalité les rayons de notre cristallin.

Nous en sommes donc arrivés au moment où une image optique est projetée sur la surface de la rétine, comme cela a lieu également dans toute camera obscura. Certes, celle-ci ne voit pas l'image, tandis que l'œil la voit. En quoi consiste cette différence? Elle consiste dans le fait que la rétine, qui, dans l'œil, reçoit l'image optique, est une partie sensible de notre système nerveux. Sous l'effet de la lumière en tant que stimulus externe, des sensations lumineuses se forment en elle. Mais que savons-nous de la manière dont la lumière provoque la sensation de lumière?

Selon l'ancienne opinion, qui était apparemment la plus naturelle, la rétine de l'œil possédait une sensibilité beaucoup plus prononcée que n'importe quel autre appareil nerveux du corps, et, pour cette raison, elle était capable de sentir le contact d'un agent même aussi fin que la lumière. La nature de l'impression produite par la lumière sur l'œil diffère entièrement, soit de la sensation des sons, soit des sensations du chaud, du dur, du mou, du rugueux, du lisse, etc., éprouvées par la peau. L'explication en était simplement que la lumière était quelque chose d'autre que le son, que la chaleur, qu'un corps dur ou mou, rugueux ou lisse. On ne trouvait rien à redire à ce que chaque chose soit ressentie différemment d'après ses qualités propres.

Il y avait cependant certains phénomènes embarrassants, que l'on tenait volontiers pour insignifiants et auxquels on préférait n'accorder aucune attention. Par exemple, si l'on presse ou frappe l'œil, des phénomènes lumineux apparaissent même dans l'obscurité la plus profonde. Des courants électriques dirigés à travers l'œil produisent eux aussi des phénomènes lumineux. À vrai dire, point n'est besoin de recourir à des moyens aussi violents ; quiconque prête attention à son champ visuel quand il ferme les yeux dans l'obscurité la plus totale, observe toutes sortes de figures curieuses, crénelées, étoilées ou striées, diversement colorées, qui se transforment sans cesse en exécutant un spectacle fantastique et déréglé. Ces apparitions deviennent plus claires et prennent des couleurs plus intenses si l'on frotte l'œil ou si des boissons excitantes, ou encore des maladies, font monter le sang à la tête. Mais elles ne font jamais défaut. On les appelle la poussière lumineuse du champ visuel obscur. 
Lorsqu'on prenait la peine d'observer ces phénomènes dans l'intention de les expliquer, on pensait que la lumière pouvait être produite dans l'œil par des processus internes. On expliquait le phénomène par une mystérieuse parenté du fluide nerveux rétinien avec la lumière : en vertu de cette parenté, la stimulation de l'un aurait provoqué l'apparition de l'autre. Les yeux luisants des chats et des chiens semblaient fournir la preuve d'une telle possibilité, puisqu'ils paraissaient produire de la lumière par eux-mêmes. Ils étaient censés luire de manière très éclatante quand on mettait ces animaux en colère, c'est-à-dire quand leur système nerveux subissait une excitation. On croyait ainsi observer dans l'œil une réelle diffusion de lumière.

Vous allez tout de suite vous rappeler la légende populaire allemande d'un partisan de cette opinion. Je songe au plus célèbre de tous les chasseurs allemands, le baron de Münchhausen, qui, après avoir perdu la pierre à feu de son fusil, se vit poursuivi par un ours. Sa présence d'esprit et sa génialité légendaire le firent trouver un expédient inattendu. Se mettant dans la position voulue, il ajusta son tir et se donna un coup de poing dans l'œil, de manière à faire jaillir des étincelles : la poudre s'alluma, l'ours était mort. Toutefois, un cas juridique a jadis suscité un embarras bien réel. Un plaignant, qui avait reçu un coup dans l'œil par une nuit noire, prétendait que la lueur provoquée par le coup lui avait permis de reconnaître son agresseur. Si la thèse physiologique que je viens de vous exposer avait été vraie, il aurait fallu tenir pour plausible la déposition de cet homme. La théorie de l'émanation lumineuse de l'œil fut donc citée à comparaître, et j'observe avec satisfaction qu'outre les arguments usuels qui la combattaient, la sentence du juge, elle aussi, a confirmé sa condamnation.

Un examen plus poussé a permis d'aborder la question d'une manière toute différente. Premièrement, la grande sensibilité supposée du nerf optique n'a nullement été confirmée : au contraire, il est apparu que la lésion du nerf optique ne provoquait pour ainsi dire aucune douleur, tandis que celle d'un autre nerf de la peau de même envergure s'accompagnait d'une douleur foudroyante. Dans quelques cas pathologiques affligeants il faut enlever le globe oculaire pour sauver la vie du malade; or, lors de la section du nerf optique, l'opéré n'éprouve aucune douleur, mais croit voir un éclair.

En outre, des enquêtes soigneusement menées ont mis en évidence, de manière unanime, que dans l'obscurité totale les yeux soi-disant luisants des félins ne brillaient absolument pas, et que leur luminosité tenait uniquement au fait qu'ils renvoient une lumière externe. En effet, on trouve à l'arrière-plan de leurs yeux non pas une matière colorante noire, mais une zone colorée claire et brillante qu'on nomme le tapis, capable de réfléchir vivement la lumière qu'elle reçoit. Plus tard, Brücke a révélé que dans un endroit convenablement éclairé, la pupille de l'œil humain, elle aussi, diffuse une lueur rouge à laquelle on peut attribuer l'apparence d'une braise, et l'emploi du rétinoscope repose précisément sur ce fait. Il est également faux qu'on puisse observer de la lumière dans l'œil d'autrui quand celui-ci perçoit des éclairs extrêmement vifs à la suite d'une pression, de courants électriques ou d'autres circonstances. 
Nous ne pouvons douter qu'en pareils cas la sensation de lumière a lieu sans qu'une lumière réelle affecte l'œil, mais nous savons que les moyens par lesquels cette sensation est provoquée (choc, pression, friction de nature mécanique ou courants électriques) suscitent toujours l'activité de l'appareil nerveux qu'ils affectent; c'est pourquoi nous les nommons moyens stimulants des nerfs. Nous pouvons par conséquent avancer ce théorème général : lorsque les stimuli nerveux communs agissent sur les nerfs oculaires, ils provoquent la sensation de lumière exactement comme la lumière réelle. Et nous pouvons ajouter, en songeant aux personnes opérées, qu'ils ne provoquent dans le nerf optique aucune autre sensation que celle de lumière.

Lorsque nous faisons agir ces mêmes stimuli sur d'autres nerfs, jamais une sensation lumineuse ne se produit : la stimulation des nerfs auditifs provoque des sensations sonores, celle des nerfs de la peau, des sensations tactiles ou encore la sensation de chaleur; la stimulation des nerfs musculaires, quant à elle, ne provoque aucune sensation mais des spasmes musculaires. C'est seulement lorsqu'ils agissent sur l'œil que ces divers stimuli provoquent la sensation de lumière. Le domaine des sensations suscitées dans le corps par le courant électrique est le plus riche : on peut facilement faire agir l'électricité sur la plupart des appareils nerveux et ainsi les stimuler très fortement. Dans l'œil, le début de la décharge électrique se signale par un éclair lumineux, suivi d'un éclairage plus doux du champ visuel, éclairage qui, suivant la direction du courant, est d'une couleur bleu clair ou bien orange. L'interruption du courant provoque à son tour un éclair. Sur la langue, le courant provoque, suivant sa direction, la sensation gustative de quelque chose d'aigre ou bien d'amer comme l'alun, sur la peau, la sensation de brûlure ou de morsure, à l'intérieur des membres, il provoque des contractions, etc.

Quel dommage, penserez-vous peut-être, que les autres centres nerveux de notre corps soient insensibles à la lumière; il serait en effet intéressant d'apprendre quelles sensations la lumière provoque ailleurs que dans l'œil. D'après nos représentations ordinaires, nous ne pouvons nous empêcher de croire que nous ressentons la lumière uniquement par l'œil, et non par la main. Mais réfléchissons un peu. Il vous paraîtra peu improbable que la lumière, à supposer qu'elle soit sentie par la main, ne susciterait pas en elle la même sorte de sensation que dans l'œil. Or, laissez tomber les rayons du soleil sur votre main. N'allez-vous pas les ressentir? «Si, répondrez-vous, je les sens bien; mais ce que je sens, c'est la chaleur du soleil, non la lumière; la chaleur est toujours associée à la lumière. » Soit, je ne vous tiens pas rigueur de cette réponse, car l'écrasante majorité des physiciens, jusqu'à ces deux dernières décennies, a répondu de la même manière. Si la lumière est toujours accompagnée de chaleur, on peut toutefois se demander si la chaleur et la lumière ne sont pas les manifestations différentes d'un seul et même principe. La physique a soigneusement examiné cette question et elle a adopté jusqu'à présent la position suivante : dans la lumière simple, monocolore, décomposable au moyen d'un prisme transparent, la puissance de réchauffement est indéfectiblement liée à celle de l'illumination. Quand l'une des deux décline, l'autre diminue 
dans la même proportion, comme il est nécessaire si chaleur et lumière ne sont que les effets du même agent. Dans les lumières de nature différente, c'est-à-dire de couleur différente, la puissance de réchauffement est liée à celle d'illumination à des degrés très divers. À luminosité égale, la lumière jaune chauffe davantage que la bleue, la rouge davantage que la jaune. Aux rayons rouges se rattachent dans le spectre solaire des rayons caloriques sombres, c'està-dire des irradiations qui émettent bien de la chaleur, mais qui n'éclairent absolument pas. Du point de vue de la seule physique, ces irradiations sont de même nature que les rayons lumineux, et elles ne s'en distinguent que par l'effet produit sur l'œil humain. Des fourneaux brûlants émettent ce genre de rayons caloriques sombres, auxquels peuvent s'associer des rayons caloriques lumineux, lorsque la température atteint le niveau du chauffage à rouge.

Ainsi, la seule différence qui subsiste entre la chaleur et la lumière résiderait dans les différentes sensations qu'elles suscitent, selon qu'elles affectent l'œil ou la peau, suscitant ici la sensation de lumière, là, celle de chaleur. Sommesnous fondés à conclure que ces effets différents correspondent à deux agents physiques différents? Pas vraiment, si nous considérons ce que j'ai dit des effets respectifs du courant électrique et de la stimulation mécanique exercée sur les différents nerfs. Le rayonnement des corps lumineux et chauds - que la physique interprète comme le mouvement ondulatoire d'une matière élastique répandue partout, à savoir l'éther —, autrement dit les ondulations de l'éther, doivent être intégrées dans la classe des stimuli nerveux. Comme tous les autres excitants, elles suscitent différentes impressions en agissant sur les différents nerfs, impressions qui relèvent respectivement du groupe de sensations inhérent à tel ou tel appareil nerveux particulier.

Nous en arrivons ainsi à la théorie des énergies sensorielles spécifiques de Johannes Müller, qui constitue le plus important progrès de la physiologie des organes sensoriels à l'époque moderne : la qualité de nos sensations, lumière, chaleur, son ou saveur, etc., ne dépend pas de l'objet externe offert à la perception, mais du nerf sensible qui transmet la sensation. Si vous êtes amateurs d'expressions paradoxales, vous pouvez dire : la lumière ne devient lumière que lorsqu'elle rencontre un æil qui voit, sans lequel elle n'est qu'une ondulation de l'éther.

Il en va presque de même pour les modifications de la sensation lumineuse, à savoir les couleurs. Les sensations des différentes couleurs sont suscitées par des ondes de différente fréquence. Les fréquences les plus courtes engendrent la sensation de violet, les plus lentes, à mesure que leur durée augmente, les sensations de bleu, vert, jaune, orange, rouge. Lorsque la lumière mêle des couleurs différentes, on a la sensation d'une couleur nouvelle, d'une couleur mixte toujours plus blanche et moins saturée que les couleurs simples qui la composent. Des couleurs mixtes d'apparence parfaitement identique peuvent cependant résulter des compositions très diverses. Leur identité n'existe alors que pour l'œil ; elle n'existe pas sous n'importe quel autre rapport physique. 
Ces faits montrent très concrètement que la qualité de nos perceptions est conditionnée autant par la nature de nos sens que par les objets externes. Ce point est de la plus haute importance pour la théorie de la connaissance. Ce que la physiologie des sens a récemment établi en suivant la voie de l'expérience correspond exactement à ce que Kant avait déjà cherché à établir à propos des représentations de l'esprit humain en général, en exposant la part que prennent les lois particulières innées de l'esprit, ainsi que son organisation, dans nos représentations. Une philosophie plus récente, en revanche, partant du présupposé d'une identité de la nature et de l'esprit, a cherché à faire de ces lois de l'esprit les lois de la réalité même. Elle a dû par conséquent tenter d'établir une équivalence entre nos impressions sensorielles et les propriétés réelles des corps perçus. Ceci l'a conduit à prendre expressément la défense de la théorie des couleurs de Goethe. J'ai tenté de montrer, en une autre occasion, que le débat concernant cette théorie tournait essentiellement autour de cette question ${ }^{1}$.

C'est donc la lumière externe qui fait naître la sensation de lumière. Cette sensation est ensuite transmise par les fibres du nerf optique jusqu'au cerveau où elle accède à la conscience. Cette sensation de lumière n'est cependant pas encore la vision. Elle ne devient vision que dans la mesure où elle nous fait connaître les objets du monde externe. Voir, c'est donc tout d'abord comprendre la sensation lumineuse. Dans le registre psychologique de notre recherche, le fait le plus remarquable qui s'offre à nous est le suivant : chaque sensation lumineuse donne lieu à la représentation de quelque chose de clair, situé en face de nous dans le champ visuel. Cela semble tout à fait simple et naturel, vu que notre sensation lumineuse est toujours suscitée par la lumière externe. Toujours? Ce n'est pas tout à fait exact. J'ai indiqué plus haut que cette sensation était également suscitée par d'autres excitations affectant le nerf optique et la rétine. Dans ces cas-là, nous avons également l'impression que la lumière provient de l'espace qui est en face de nous. Gardons les yeux ouverts, et recevons du front jusqu'à la nuque un courant électrique qui excite également notre nerf optique : alors même que le courant électrique ne produit en l'occurrence aucune lumière objective, aucune ondulation de l'éther, ni dans notre oil ni dans le monde externe, nous croyons cependant voir un éclair lumineux qui illumine les corps situés en face de nous. Dans ce cas-là, la sensation de lumière devient une illusion sensorielle. Toutefois, ce n'est pas l'organe sensoriel qui nous trompe. Son action n'est nullement déréglée; au contraire, il agit d'après ses lois fixes et immuables et ne peut absolument pas agir autrement. C'est nous qui nous méprenons dans la compréhension de l'impression sensorielle.

En outre, l'excitation d'une zone déterminée de la rétine suscite la représentation d'un corps lumineux dans un lieu déterminé de l'espace situé devant nous. Comme je vous l'ai exposé auparavant, lorsque la vision est nette, la lumière en provenance d'un point déterminé du champ visuel ne touche qu'un

1. Voir ma contribution sur les travaux scientifiques de Goethe. 
seul point de notre rétine. C'est pourquoi nous situons toujours l'origine de toute sensation lumineuse survenant en ce point de la rétine dans l'endroit correspondant du champ visuel. Or, si vous pressez avec votre ongle le coin extérieur de votre œil, un petit reflet lumineux apparaît. Dans un premier temps, vous ne le remarquerez peut-être pas du tout, car vous le cherchez là où vous pressez. Grave erreur! Il apparaît précisément du côté opposé du champ visuel, à proximité du dos du nez, sous l'aspect d'un petit cercle clair. Si vous pressez l'œil sous la bordure du sourcil de l'orbite, le reflet lumineux apparaîtra dans la zone de la paupière inférieure. Bref, de quelque côté de l'œil que vous exerciez la pression, le reflet lumineux apparaît toujours du côté opposé.

L'explication du phénomène découle déjà de ce qui vient d'être dit. Revenons au cas où nous pressons le coin extérieur de l'œil et où le reflet lumineux apparaît au dos du nez. La pression excite alors les points de la rétine qui ont coutume de recevoir la lumière issue du dos du nez. En effet, l'image sur la rétine est inversée, et l'image du dos du nez est projetée sur la face extérieure de la rétine. Si nous nous laissons aller à inférer, de l'excitation de la rétine par la pression de l'ongle, à la présence effective de lumière, il est tout à fait logique que nous situions l'origine de cette lumière dans le même lieu de l'espace que celui d'où provient la lumière réelle lorsqu'elle touche l'endroit en question de la rétine. Dans l'expérience de toute notre vie passée, nous avons cherché et trouvé au dos du nez l'origine de la lumière qui touche les parties extérieures de la rétine, et pareillement notre représentation situe spontanément au même emplacement la lumière apparente due à la pression. Cette manière de se représenter les choses nous paraît naturelle et compréhensible. Je voudrais cependant attirer l'attention sur une circonstance frappante, propre à susciter l'étonnement.

Nous venons de prendre notre représentation en flagrant délit de faux raisonnement; nous avons compris scientifiquement ce qui la poussait à la faute, et comment les choses se passaient en réalité. Nous savons que notre représentation a fait une inférence qui s'est vérifiée des millions de fois, mais qu'elle est fausse dans un cas, auquel elle ne s'applique pas. À présent, notre représentation devrait facilement reconnaître ce qu'il en est et ne plus nous abuser par la fausse image d'un reflet lumineux dans la zone du dos du nez. Elle devrait au contraire situer le reflet lumineux là où la sensation lumineuse est effectivement provoquée. Nous réitérons l'expérience, cette fois avec la conviction scientifiquement assurée que la sensation lumineuse survient dans le coin extérieur de l'œil. Notre représentation s'est-elle laissé corriger? Nous devons reconnaître que non. Elle se comporte comme le plus cancre de tous les écoliers : elle laisse parler sa malheureuse maîtresse, Madame la science, tant qu'elle le veut, et maintient avec entêtement que le reflet lumineux se situe dans la zone du dos du nez.

Il en va de même pour toutes les illusions sensorielles dont je vais vous parler. Une fois leur mécanisme découvert, elles ne disparaissent pas, mais persistent avec une force inépuisable. 
Une autre série d'illusions sur l'emplacement de l'objet vu se produit lorsque la lumière, qui parvient jusqu'à l'œil non sans certaines altérations de l'objet, s'est heurtée à des corps miroitants ou réfractant. Le cas le plus connu de cette espèce est la réflexion sur les miroirs plans ordinaires. La lumière qui tombe sur le miroir est renvoyée par lui de telle manière qu'elle nous semble provenir d'objets situés loin derrière la surface du miroir, d'aussi loin que le sont les objets réels situés devant cette surface. Lorsque l'image réfléchie atteint l'œil, elle arrive naturellement dans celui-ci tout aussi réfractée, et elle touche les mêmes points de la rétine que ceux que toucherait la lumière issue des corps réels s'ils se trouvaient à l'endroit apparent des images optiques du miroir. Notre représentation sensible s'invente ainsi une construction des corps réels qui correspondent aux images du miroir : elle leur confère le même degré de détermination et d'évidence qu'aux corps vus de manière directe. Là encore, nous affirmons que la vivacité et la position apparente de l'image demeurent entières, quoique notre entendement sache qu'il n'y a là aucun objet.

Il en va de même avec la lunette d'approche et le microscope. Dans leurs verres respectifs, la lumière est réfractée de telle manière que lorsqu'elle a franchi l'instrument, elle poursuit sa course comme si elle provenait d'un objet amplifié, et l'observateur qui regarde à travers l'instrument croit réellement voir cet objet amplifié.

D'après l'endroit de notre rétine où la sensation lumineuse se produit, nous jugeons dans quelle direction se trouvent les différents objets clairs qui nous entourent, et dans quelles parties du champ visuel nous devons les situer. Nous obtenons ainsi une image perspective du monde extérieur, l'image optique sur notre rétine formant déjà une image perspective. Une image perspective correcte des objets possédant une forme régulière, bien connue de nous, nous permet également de former un jugement assez juste sur les dimensions de profondeur des objets représentés, surtout quand de surcroît le jeu d'ombre et de lumière est approprié. De bons dessins perspectifs de pièces de machine, d'édifices, etc., nous suffisent, car nous savons que le dessin est censé représenter des objets dont la forme fondamentale est régulière, sphérique, cylindrique ou prismatique. Dans le cas d'objets à forme irrégulière, en revanche, les dessins perspectifs ne donnent qu'une intuition très imparfaite de la dimension de profondeur. Dans la peinture de paysage intervient en outre ce qu'on appelle la perspective de l'air : la couleur et la clarté des contours sont altérées par des couches d'air interposées. Or, qu'est-ce qui distingue un dessin perspectif de l'aspect de l'objet lui-même, tel que nos yeux nous l'offrent directement, et pourquoi reconnaissons-nous beaucoup mieux et beaucoup plus sûrement les données corporelles lorsque nous les voyons de manière directe?

La réponse nous est donnée par un instrument d'optique qui, ces derniers temps, est devenu un objet courant de divertissement populaire : le stéréoscope, inventé par le physicien anglais Wheatstone. Cet instrument donne aux dessins perspectifs une très grande vivacité de vue en profondeur, et cela même aux dessins qui ne permettent absolument pas de juger, par un examen direct, 
quelles parties sont censées être devant, lesquelles derrière, et quelle distance sépare telle partie et telle autre située derrière.

Les principes du stéréoscope sont simplement les suivants : lorsque nous regardons une chose, un paysage, une chambre, ou quelque chose de semblable dont les différentes parties sont à des distances différentes, nous obtenons, selon différents points de vue, des visions différentes. Les objets du premier plan qui, observés selon un premier point de vue, cachaient telle zone déterminée de l'arrière-plan, cachent selon un second point de vue des zones différentes. Les surfaces qui, selon un premier point de vue, semblaient fortement réduites, semblent l'être moins selon un second, et inversement. Ainsi, lorsque, à partir de deux points de vue différents, nous obtenons des visions perspectives d'un même objet corporel étendu, ces visions ne se ressemblent pas, mais diffèrent autant que les points de vue en question. Par contre, le dessin perspectif le plus parfait ne subit aucune altération importante quant à son aspect si nous regardons successivement ce dessin de deux points de vue différents. Les objets au premier plan du dessin cachent toujours exactement les mêmes zones de l'arrière-plan, et les surfaces qui, au premier coup d'œil, semblaient réduites, le restent.

Or, l'homme a deux yeux qui regardent continuellement le monde de deux points de vue différents, et qui, chaque fois qu'ils perçoivent un objet corporel étendu, offrent continuellement deux vues perspectives différentes à la conscience pour qu'elle les juge. En revanche, lorsque les deux yeux contemplent le dessin perspectif d'un objet réalisé sur une surface plane, tous deux reçoivent la même image perspective. C'est grâce à cette différence que nous sommes en mesure de distinguer l'objet réel de sa copie, aussi fidèle et parfaite soit-elle.

Mais supposons que nous réalisions deux dessins perspectifs du même objet, l'un correspondant à ce que voit l'œil droit, l'autre à ce que voit l'œil gauche. Présentons ensuite à chaque œil, dans une position convenable, le dessin qui lui correspond. À présent, la différence essentielle entre la vision de l'original et celle de sa copie disparaît, et nous croyons voir des objets réels en lieu et place des dessins.

Telle est la prestation fournie par le stéréoscope. Les représentations stéréoscopiques comprennent toujours deux dessins du même objet, formés à partir de deux points de vue différents. La partie optique de l'instrument peut être construite de manière extrêmement diverse, mais toute sa fonction consiste à donner l'impression que les deux dessins différents sont situés au même endroit. À vrai dire, celui qui sait loucher n'a nullement besoin d'expédients optiques de ce type. Disposons simplement les deux dessins l'un à côté de l'autre et louchons jusqu'à ce que les deux images médianes de ces images dédoublées se recoupent : l'illusion stéréoscopique se produit alors.

À cet égard, les présentations stéréoscopiques de figures corporelles composées de simples lignes et de points sont les plus riches d'enseignement. Ici, tous les autres moyens servant à juger la dimension de profondeur font défaut, si bien que l'illusion et son principe apparaissent de manière très évi- 
dente. Pour l'extraordinaire vivacité de l'illusion engendrée, les représentations stéréoscopiques initialement conçues et réalisées par $\mathrm{Moser}^{2}$ sont les plus admirables : ce sont des représentations de paysages, de statues et de figures humaines obtenues au moyen de la photographie.

Ainsi, à partir des deux vues perspectives distinctes fournies par nos deux yeux, nous construisons continuellement les rapports spatiaux entre les objets de notre entourage. Le borgne est privé de cet avantage; tant qu'il ne bouge pas, il reconnaît son entourage avec autant de justesse que s'il avait affaire à sa peinture parfaitement fidèle. C'est en se déplaçant qu'il obtient des vues à partir de différents points de vue, et qu'il apprend à bien juger les rapports spatiaux. On peut donc dire que tant qu'il ne bouge pas, il ne voit pas le monde à proprement parler mais seulement un tableau perspectif du monde. Il ne peut non plus tirer aucun avantage du stéréoscope, puisque l'illusion procurée par l'usage de cet instrument repose sur l'usage simultané des deux yeux.

On explique de la même manière la difficulté d'un jeu pratiqué de temps à autre par la jeunesse. On suspend un anneau à un fil. L'un des joueurs se positionne de telle manière qu'il voit l'anneau de biais. L'œil fermé, il a pour tâche de passer une baguette à travers l'anneau. Habituellement, non sans provoquer les rires, il n'y parvient qu'après maintes tentatives infructueuses. Les deux yeux ouverts, en revanche, la tâche est très facile à accomplir.

Il faut évoquer un dernier élément dont nous avons besoin pour évaluer les rapports spatiaux. Nous jugeons de quelle direction vient la lumière d'après la position de la rétine touchée par elle. Toutefois, la position de l'image rétinienne se modifie avec le déplacement de l'œil. Il faut donc connaître la position de l'œil dans notre tête si nous voulons inférer correctement les rapports spatiaux. Chaque mouvement de l'œil qui ne procède pas de notre action volontaire ou qui ne s'accomplit pas comme voulu perturbe de ce fait notre jugement sur la situation des objets qui nous entourent. Si on ferme un œeil en pressant et en étirant l'autre, les objets vus semblent se mouvoir. Du fait de la pression mécanique externe, l'œil est alors déplacé sans que nous puissions exactement juger dans quelle direction et dans quelle proportion ; à cette occasion, les images optiques se déplacent sur la rétine, et nous en inférons que les objets se meuvent. Si nous faisons cette expérience les deux yeux ouverts, l'œil immobile perçoit l'objet comme ferme et fixe, tandis que l'œil pressé ou étiré voit une seconde image adjacente et mobile du même objet. Les mouvements apparents causés par le vertige sont du même ordre. Ils s'expliquent en grande partie par le fait que l'action des muscles qui font mouvoir l'œil est mal jugée. Un fiévreux à qui les objets paraissent danser lorsqu'il bouge les yeux, et ce parce qu'il juge mal de l'action de ses muscles oculaires sur la position de son œil, ressent un soulagement dès qu'il maintient ses yeux en une seule position en se fixant sur un point : alors, la cause des mouvements apparents disparaît. Quelqu'un qui, après s'être tourné rapidement sur lui-même, s'immobilise, voit les objets dans son environnement accomplir un mouvement apparent dans le

2. Voir Dove, Repertorium der Physik, Volume V, 1844, 384. 
sens opposé. Celui qui, assis dans un train, regarde à l'intérieur du wagon après avoir longtemps contemplé les objets devant lesquels le train passait, croit voir les objets du wagon se déplacer dans la direction opposée. Celui qui a navigué un certain temps en mer, une fois à terre, croit voir sa chambre bouger de la même manière que la cabine du navire. Dans les cas de ce genre, une habitude de mal juger s'est développée. Pendant toute la durée du déplacement réel d'un objet, l'observateur devait, pour fixer l'objet, accompagner ce déplacement de ses yeux. S'est ainsi instaurée en lui une nouvelle forme d'exercice, qui lui a appris quel degré de tension il devait donner aux muscles oculaires pour fixer un objet. Or, si le mouvement réel prend fin, l'observateur tend à fixer sans relâche les objets. Mais à présent, en imprimant la même tension aux muscles, l'image rétinienne se décale, car les objets immobiles ne se déplacent plus avec les yeux. L'observateur en infère que les objets immobiles se meuvent, jusqu'à ce qu'il réussisse à nouveau à fixer des objets immobiles. Cette sorte de mouvements apparents nous intéresse parce qu'ils montrent la rapidité avec laquelle une modification peut survenir dans le travail d'interprétation des perceptions sensorielles.

Voici quelques autres exemples montrant combien, dans l'usage pratique quotidien que nous faisons de nos organes sensoriels, nous songeons peu au rôle qu'ils jouent dans cet usage. En effet, la seule chose qui nous intéresse dans les perceptions fournies par ces organes, c'est ce qu'elles nous apprennent des états du monde externe, au point que nous négligeons les perceptions qui ne remplissent pas cette fonction. Quand nous voulons considérer exactement un objet, nous dirigeons nos yeux sur lui de manière à le voir clairement en tant que tel. Si ensuite, en exerçant une pression avec le doigt, nous déplaçons l'œil de côté, surgissent alors, comme je l'ai mentionné, des images doubles de l'objet, parce qu'à présent nous ne situons plus les images des deux yeux au même endroit du champ visuel. Pendant que nous fixons l'objet, la position de nos yeux n'est pas plus adaptée à l'ensemble des objets plus proches ou plus lointains que l'objet fixé : en effet, tous ces objets nous apparaissent en double.

Tenons un doigt juste devant nos yeux. Si l'on regarde ce doigt tout en considérant les objets à l'arrière-plan, ceux-ci apparaissent en double; par contre, si on regarde un point de l'arrière-plan, c'est le doigt qui apparaît en double. Nous ne pouvons donc pas douter que nous voyons durablement en double la majeure partie des objets du champ visuel, bien qu'il ne soit vraiment pas facile de s'en apercevoir. Peut-être de nombreuses personnes ne s'en sont-elles jamais aperçu de toute leur vie. Et même si nous sommes conscients de ce fait, un effort particulier d'attention est toujours nécessaire pour percevoir les images dédoublées, car l'usage pratique habituel de nos organes visuels s'entête à faire abstraction de ces images, même quand elles persistent avec le plus de force.

En outre, croirez-vous qu'il y a dans chaque œil humain un emplacement, tout proche du milieu du champ visuel, qui ne voit absolument rien? Cette zone parfaitement aveugle correspond à l'entrée du nerf optique. Et ce 
fait échapperait à notre observation toute notre vie durant? Comment est-ce possible? Pour le comprendre, supposons le cas d'une personne aveugle d'un œil depuis des mois ou des années, et qui n'en prendrait conscience qu'à l'occasion d'une indisposition accidentelle de son autre œil. C'est le même phénomène qui a lieu avec la tache aveugle de l'œil normal. Les taches aveugles des deux yeux ne se situent pas dans la même partie du champ visuel. Ainsi, chaque œil voit quelque chose que l'autre ne peut pas voir. Et même quand nous fermons un œil, il n'est pas si facile de prendre conscience de la tache qui devient subitement aveugle, car d'ordinaire, pour voir quelque chose avec netteté, nous n'utilisons qu'un seul lieu de la rétine (lieu d'une constitution particulière remarquable), celui de la vision directe. Les impressions provenant des autres parties du champ visuel n'offrent somme toute qu'une première ébauche de ce qui est voisin de l'objet directement contemplé. Et comme le plus souvent nous laissons notre regard errer sur les diverses parties du champ visuel, en fixant à tour de rôle les objets qui nous intéressent, ces mouvements nous permettent, en dépit de la tache aveugle, de prendre connaissance de toutes les parties du champ visuel, et rien ne nous empêche de percevoir tout de ce qui peut intéresser notre perception.

Pour se convaincre de l'existence de cette tache aveugle, il est nécessaire de procéder à quelques observations méthodiques. Fermons l'œil gauche, plaçons une feuille de papier à 7 pouces devant notre œil droit et fixons n'importe quel point de la surface du papier : la tache aveugle correspond à l'emplacement du papier situé à 2 pouces à droite du point de mire. Si une tache noire ou n'importe quel petit objet se trouve à cet endroit, on ne le voit pas : au contraire, la surface blanche du papier semble ininterrompue ${ }^{3}$.

La grandeur de la tache aveugle est suffisamment importante pour qu'elle puisse masquer, dans le ciel, un disque dont le diamètre serait douze fois supérieur à celui de la Lune. Elle peut masquer un visage humain à une distance de 6 pieds. Vous voyez que sa grandeur, toute proportion gardée, n'est nullement insignifiante.

Ces faits corroborent ce que j'ai affirmé précédemment, à savoir que dans l'usage spontané que nous faisons de nos sens, nous tenons compte de ce qui nous renseigne sur le monde extérieur. Or, des recherches récentes sur la tache aveugle nous offrent en outre des informations intéressantes sur le rôle que les processus psychiques jouent déjà au niveau des perceptions sensorielles les plus simples. Fixons, à l'endroit du champ visuel qui correspond à la tache aveugle, un objet quelconque plus petit qu'elle. Nous ne voyons pas cet objet, mais nous comblons cette lacune avec la couleur du fond où il s'inscrit, exactement

3. Le lecteur pourra facilement en faire l'expérience sur la figure suivante :

\section{$+$}

Fermez l'œil gauche, fixez la croix avec l'œil droit, et placez la feuille de papier à environ 7 pouces de votre œil : le cercle noir disparaît. Si vous rapprochez le papier ou si vous l'éloignez davantage de l'œil, le cercle réapparaît. Il faut cependant veiller à fixer continuellement la croix. 
comme dans l'expérience précitée de la tache noire sur papier blanc. Lorsque la zone aveugle concerne une partie d'une figure quelconque, nous complétons cette figure en fonction de ce qui appartient aux figures du même genre le plus souvent rencontrées. Par exemple, si la tache aveugle concerne une portion de ligne noire sur fond blanc, l'imagination prolonge cette ligne, en suivant le chemin le plus court, à travers la tache aveugle. Et elle procède de la sorte même si, à cet endroit, la ligne réelle présente en vérité un espace vide ou décrit une courbure.

Si la tache aveugle concerne le centre d'une croix, l'imagination comble la partie médiane, et nous croyons voir une croix même si en réalité les quatre branches ne se rejoignent pas au milieu, etc.

Quand des interprétations différentes nous sont aussi familières les unes que les autres, la représentation oscille souvent entre l'une et l'autre, sans que la volonté ne puisse lui imposer le choix de l'une d'elles.

Lorsque les deux yeux sont ouverts, nous nous conformons en général aux perceptions de l'œil qui voit. Si je tiens devant moi un papier comportant une tache rouge qui n'est pas vue par l'œil droit, elle est néanmoins vue par l'œil gauche. C'est pourquoi je crois percevoir un papier avec une tache rouge, ce qui est effectivement le cas. Dans d'autres cas, par contre, nous ne nous décidons pas nécessairement d'après les perceptions de l'œil qui voit. Si je prends à présent une feuille de papier tout blanc et que je tiens devant mon œil gauche un verre rouge, la feuille de papier tout entière apparaît uniformément rougeâtre, sans que l'endroit correspondant à la tache aveugle de l'œil droit ne se distingue par un aspect particulier. Et pourtant, les sensations immédiates se rapportant à cet endroit sont pour les deux yeux exactement les mêmes que dans le cas précédent, où l'on contemplait une feuille de papier marquée d'une tache rouge. En effet, l'œil droit ne voyait absolument rien, tandis que le gauche voyait un rouge pur. Dans le cas présent, en revanche, la zone aveugle correspondant à l'œil droit n'apparaît pas comme un rouge pur. Comme tout le reste du papier, elle apparaît blanche avec une faible nuance de rouge. La différence tient seulement au fait qu'à présent ce n'est pas seulement un emplacement particulier qui apparaît rouge pur à l'œil gauche, mais la surface entière du papier. Pour l'œil gauche, l'emplacement qui correspond à la zone aveugle de l'œil droit ne se distingue pas, par sa couleur, du reste du papier ; aussi le jugement n'a-t-il aucune raison d'attribuer une qualité particulière à cet emplacement. Nous avons donc là un cas où l'imagination, bien qu'elle ait affaire à des sensations similaires, est conduite par le jugement à leur donner une valeur différente, et ce à juste titre.

Le champ de ce qu'on nomme les illusions optiques est immensément riche. Toutefois, les exemples avancés suffiront à vous faire saisir concrètement les caractéristiques de nos perceptions visuelles, voire de nos sens en général.

J'ai toujours affirmé jusqu'ici que la représentation en nous jugeait, inférait, réfléchissait, etc., en me gardant bien de dire que c'est nous qui jugeons, inférons, réfléchissons. En effet, j'ai reconnu que ces actes se faisaient à notre 
insu et qu'ils ne pouvaient être infléchis par notre volonté ou notre conviction, si forte soit-elle. Est-il toutefois légitime de parler ici d'authentiques processus de pensée, alors que cette pensée échappe à la conscience et au contrôle de l'intelligence consciente de soi ? En outre, nous hésiterions à attribuer à nos inférences l'exactitude et la précision avec lesquelles se construisent nos images visuelles si adéquatement, si instantanément et si indubitablement. En effet, quelque fierté que nous tirions de la puissance de notre entendement, nous avons trop tendance, dans la vie courante et dans l'exercice des disciplines non mathématiques, à recouvrir du terme d'inférence ce qui n'est en réalité qu'une supposition ou une conjecture vraisemblable, de sorte que nous devrions douter de la fiabilité des inférences qui ne reposent pas sur l'expérience. Même quand nous pouvons atteindre une fiabilité absolue, comme dans les inférences et les calculs en mathématiques, ces opérations sont tellement laborieuses, compliquées et lentes, qu'elles sont à mille lieues de pouvoir soutenir la comparaison avec les aperceptions instantanées de l'œil.

C'est une tâche difficile que de déterminer la nature des processus psychiques qui convertissent la sensation lumineuse en une perception du monde externe. Malheureusement, nous ne trouvons aucune aide auprès des psychologues, car en psychologie le seul chemin menant à la connaissance a été jusqu'à présent l'introspection. Or, nous avons affaire ici à des actes mentaux sur lesquels l'introspection ne nous fournit aucune information. Au contraire, c'est l'analyse physiologique des appareils des sens qui nous oblige à en supposer l'existence. C'est pourquoi les psychologues ont très souvent mis ces actes mentaux immédiatement au compte de la perception sensible, sans chercher à en savoir davantage à leur sujet.

Ceux qui hésitaient à accorder un rôle à la pensée et aux inférences dans les perceptions sensibles crurent tout d'abord que la conscience quittait l'œil, qu'elle se propageait le long du rayon lumineux jusqu'à l'objet vu et qu'elle percevait celui-ci en son lieu et place, à peu près comme le décrit Platon :

Parmi les organes, c'est d'abord les porte-lumière qu'ils [les dieux] ont construits, les yeux; voici, pour nous les attacher, quelles furent leurs causes. Cette sorte de feu qui n'a pas la capacité de brûler, mais celle de procurer une douce lumière, ils ont su faire qu'elle devînt chaque jour un corps à nous approprié. En effet, le feu qui est au-dedans de nous et qui est frère de celui-là, dans sa toute pureté ils ont fait qu'il rayonne par les yeux; pour cela, ils ont rendu lisse et dense l'œil tout entier, et, plus particulièrement en son milieu, ils en ont resserré le tissu, en sorte qu'il fût étanche à tout contenu plus épais, et qu'un tel feu seulement, en sa pureté absolue, pût filtrer au travers. Lors donc qu'il y a la lumière du jour tout autour du rayon visuel, alors celui-ci s'épanche semblable vers son semblable et se combine avec lui; un corps unique, approprié au nôtre, se constitue tout le long de la droite issue des yeux, en quelque direction que le feu jailli de l'extérieur 
aille buter contre celui qui arrive des objets extérieurs. Susceptible des mêmes impressions d'un bout à l'autre en raison de son homogénéité, quel que soit l'objet dont il prenne lui-même contact ou qui vienne en contact avec lui, il en transmet les mouvements dans tout le corps jusqu'à l'âme, et lui procure cette sensation grâce à laquelle nous déclarons voir. Mais, quand se retire dans la nuit son parent, le feu extérieur, il se trouve coupé d'avec lui; rencontrant, au sortir de l'œil, un milieu dissemblable, il est altéré lui-même et s'éteint; il ne peut plus confondre sa nature dans l'air environnant, puisque celui-ci ne contient pas de feu ${ }^{4}$.

Ce qui est remarquable dans ce passage, c'est que le rôle prépondérant joué par l'œil dans la sensation de lumière est reconnu. Dans ce sens, on peut comparer la lumière interne à l'activité nerveuse. Selon Platon, en effet, la lumière interne s'épanche, se mélange à la lumière externe des corps éclairés et fait émerger la conscience de la présence du corps concerné. De la même manière, certains auteurs modernes ont soutenu qu'un mystérieux agent nerveux coulait de l'œil pour connaître les corps à leur contact. Cette doctrine fut propagée notamment par les tenants du magnétisme animal, qui construisaient leur théorie sur l'existence supposée d'une atmosphère nerveuse entourant le corps humain. Comme on sait, ils faisaient voyager le fluide nerveux jusqu'aux parties les plus éloignées de la terre et même de l'univers, pour qu'il y découvre ce que le magnétiseur curieux souhaitait savoir. Bien que cette conception de la vision puisse sembler mieux correspondre à l'expérience sensible de la vie ordinaire, elle est toutefois intenable. En effet, pourquoi le principe nerveux diffus ou la conscience ne remarque-t-elle pas, le cas échéant, que c'est le doigt seul qui a pressé la rétine, et qu'il n'y a en réalité aucune lumière externe? Et qu'advient-il de ce principe, lorsqu'à l'extérieur il rencontre un miroir ? La conscience est-elle réfléchie par lui selon les mêmes lois que la lumière? Pourquoi se laisse-t-elle alors abuser sur l'emplacement du corps vu dans le miroir? Â suivre cette hypothèse, nous nous empêtrons dans les pires absurdités; c'est pourquoi elle n'a jamais été admise dans la science digne de ce nom.

Si la conscience ne perçoit pas les corps immédiatement au lieu où ils se trouvent, elle ne peut les connaître qu'au gré d'une inférence. En effet, seules les inférences nous font connaître ce que nous ne percevons pas de manière directe. Nous sommes tous d'accord que cette inférence ne se fait pas de manière consciente. Elle se caractérise bien plutôt par son exécution mécanique, et elle entre dans la classe des associations d'idées involontaires qui naissent de ce que deux représentations s'étant présentées très fréquemment l'une avec l'autre, l'une suscite immanquablement l'autre selon une certaine nécessité naturelle. Songez à un acteur habile, qui présenterait avec fidélité la tenue vestimentaire, les gestes et les mœurs du personnage qu'il incarne. Nous aurons beau pouvoir nous dire à chaque moment que ce que nous voyons sur scène

4. NdT : Platon, Timée, $45 \mathrm{~b}-\mathrm{d}$, dans la traduction de Joseph Moreau, in : Euvres complètes de Platon, tome 2, Paris : Gallimard, 1950, 462-463. 
n'est pas le personnage qui correspond au rôle, mais l'acteur X que nous avons déjà vu jouer d'autres rôles. Toutefois, cette idée, acte de la pensée libre et consciente, n'effacera pas l'illusion qui maintient vivante en nous, durablement, l'idée que nous sommes en face du personnage lui-même. Involontairement et durablement, nous attribuerons à la personne sur scène les sentiments qui correspondent au rôle qu'elle joue, et nous nous attendrons à ce qu'elle agisse d'après ces sentiments. En effet, lorsque la représentation dramatique atteint sa plus haute perfection, nous ne pensons plus du tout à l'art de l'acteur, car ce qu'il fait nous semble tout naturel. Nous apprenons à apprécier un acteur de premier ordre en le comparant à ses partenaires moins habiles, qui nous rappellent sans cesse notre illusion par certains traits qui appartiennent à leur personnalité d'acteur et non au personnage incarné.

Il en va exactement de même avec les illusions optiques, une fois que nous pénétrons leur mécanisme. Dans ce genre de cas, même si nous savons que la représentation suscitée en nous par l'impression sensible est fausse, elle n'en persiste pas moins dans toute sa vivacité. Certes, l'illusion est entretenue chez l'acteur uniquement par des formes conventionnelles touchant l'habillement, les gestes, la manière de parler. C'est tout au plus face aux épanchements de la passion que nous pouvons concevoir une connexion naturelle entre les sentiments et les signes qu'en donne l'acteur. Dans les perceptions sensorielles en revanche, nous avons affaire à une connexion d'idées conditionnée par la nature même de nos sens, et qui admet beaucoup moins d'exceptions que la connexion d'idées faisant intervenir les mœurs humaines dans toutes leurs formes. Toute notre vie durant, nous avons expérimenté des millions de fois que quand un objet suscite telle sensation lumineuse dans certaines fibres nerveuses de nos deux yeux placés d'une certaine manière, nous devions tendre le bras jusqu'à une certaine distance ou faire un nombre déterminé de pas pour l'atteindre. S'est ainsi formée en nous une association involontaire entre une impression visuelle déterminée et la distance et la direction selon lesquelles nous devions chercher l'objet. Cette association explique que la représentation d'un objet se forme et se maintienne même quand c'est un stéréoscope, par exemple, qui suscite en nous les impressions visuelles adéquates, et ce malgré notre conviction parfaitement fondée qu'il n'y a en réalité aucun objet. Exactement de la même manière, les vêtements et mouvements de l'acteur maintiennent vivace notre intuition du personnage joué par l'acteur. Dans ce dernier cas toutefois, l'association entre l'aspect extérieur et la nature du personnage, par exemple entre les vêtements virils et le genre masculin, est purement conventionnelle. Elle n'est pas donnée en vertu d'une nécessité naturelle; elle n'est donc pas innée mais ne peut être qu'acquise. Quant à l'évaluation de la distance au moyen des yeux, nous ne pouvons pas douter qu'elle s'acquiert elle aussi par un exercice répété. L'observation de jeunes enfants nous révèle que ceux-ci véhiculent des représentations très erronées de la distance des objets qu'ils aperçoivent, et plusieurs d'entre vous pourront peut-être se rappeler de cer- 
taines circonstances de leur enfance où ils se sont grossièrement trompés sur les distances. Je garde encore nettement à l'esprit le jour où j'ai compris la loi de la perspective, qui veut que les objets éloignés paraissent petits. Je passais à côté d'une haute tour, sur la galerie supérieure de laquelle se trouvaient des hommes, et je demandais à ma mère de me descendre ces jolies poupées, étant persuadé qu'il lui suffisait de tendre le bras pour atteindre la galerie de la tour. Plus tard, j'ai souvent levé les yeux vers cette galerie quand des hommes s'y trouvaient, mais ceux-ci, auprès de mon œil exercé, ne passaient plus pour de jolies petites poupées.

L'idée d'un exercice répété et d'une éducation de nos organes sensoriels permet également d'expliquer la sûreté et la précision avec lesquelles nos yeux construisent l'espace. La précision avec laquelle nous apprenons à utiliser les organes de notre corps dépasse celle des machines artificielles, comme en attestent les exercices du jongleur ou les coups des joueurs de billard éprouvés. En quelque sorte, nous sommes tous jongleurs des yeux; nous nous sommes tous exercés de manière beaucoup plus continue et durable à évaluer nos objets visuels que notre artiste gymnaste aux jeux de quilles et d'équilibre; et si notre art ne fait pas sensation, c'est uniquement parce que chacun peut réaliser les mêmes tours d'adresse que les autres.

Apprendre à voir se réduit pour nous à apprendre à associer à certaines sensations perçues par nous la représentation d'un objet déterminé. Dans cette affaire, les éléments intermédiaires grâce auxquels les sensations se produisent ne nous intéressent pas du tout; sans procéder à un examen scientifique, nous n'en saurions rien. L'image optique sur la rétine fait, elle aussi, partie de ces éléments intermédiaires. Le fait que nous voyions les objets à l'endroit, alors que cette image est à l'envers, a suscité beaucoup d'étonnement et une infinité de tentatives d'explication inutiles. L'expérience nous a appris que dans certaines fibres du nerf optique, la sensation lumineuse indiquait les objets lumineux comme étant en haut, et, dans certaines autres, comme étant en bas. Que ces fibres soient situées dans la rétine et dans le nerf optique n'a aucune importance, du moment que nous sommes en mesure de distinguer l'impression d'une fibre de l'impression d'une autre. Qu'il existe une rétine où se trouvent des images optiques, l'homme naïf l'ignore complètement. Comment la position de l'image optique sur la rétine pourrait-elle l'induire en erreur?

Quelle part les associations d'idées entièrement acquises prennent-elles dans l'interprétation de nos perceptions sensorielles, par rapport aux associations d'idées innées, donc conditionnées par l'organisation de l'homme? Jusqu'à ce jour, on ne sait quasiment pas répondre à cette question. Nous observons toutefois chez les bêtes des comportements instinctifs qui nous instruisent à ce sujet. Le veau nouveau-né se dirige vers le pis de la vache pour téter ; s'il le faisait consciemment, cela voudrait dire qu'il comprend les phénomènes visuels et qu'il connaît l'usage de ses pieds, or il n'a pu apprendre ni l'un ni l'autre. Mais qui d'entre nous peut se projeter dans l'âme d'un veau qui vient de naître pour comprendre le mécanisme de ce comportement instinctif? 
Est-il donc concevable que ce que j'ai précédemment nommé l'acte de penser et d'inférer ne relève en réalité ni de la pensée ni de l'inférence, mais d'une simple association d'idées s'effectuant de manière mécanique? Je vous prie de faire encore un dernier pas avec moi, un pas qui nous ramènera à Kant, notre point de départ. Pour pouvoir associer à certaines sensations la représentation d'un corps présentant tel aspect et occupant tel lieu, nous devons préalablement avoir une notion du genre de corps dont il s'agit. Il en va d'ailleurs ainsi de l'œil comme des autres sens : jamais nous ne percevons immédiatement les objets du monde extérieur, nous en percevons seulement leurs effets sur nos appareils nerveux, et ce, dès les premiers instants de notre vie. De quelle manière sommes-nous donc passés du monde des sensations nerveuses au monde de la réalité? Manifestement, au seul gré d'une inférence. Nous devons présupposer l'existence d'objets extérieurs comme cause des excitations nerveuses, car il ne peut y avoir d'effet sans cause. D'où tirons-nous le savoir qu'il n'y a pas d'effet sans cause? S'agit-il là d'un principe de nature empirique? Certains ont voulu le faire passer pour tel. Or, nous voyons bien que nous avons besoin de ce principe avant même d'acquérir quelque connaissance que ce soit des choses du monde externe. Nous en avons besoin ne serait-ce que pour parvenir à savoir qu'il y a des objets autour de nous dans l'espace, objets entre lesquels un rapport de cause à effet peut exister. Pouvons-nous tirer ce principe de l'expérience interne que nous faisons de la conscience de soi? Non, car nous considérons les actes de notre volonté et de notre pensée, par lesquels nous prenons conscience de nous-mêmes, comme des actes libres. Nous nions qu'ils soient les effets nécessaires de causes suffisantes. Ainsi, l'examen des perceptions sensorielles nous ramène à cette découverte de Kant : le principe selon lequel il n'y a «pas d'effet sans cause» est une loi de notre pensée donnée avant toute expérience.

La philosophie a accompli un progrès extraordinaire avec Kant, qui a mis au jour le principe de causalité et les autres formes innées de l'intuition et de la pensée en démontrant leur existence. Ce faisant, comme je l'ai signalé précédemment, il a établi pour la théorie des représentations en général l'équivalent de ce qu'en physiologie Johannes Müller a établi par des méthodes empiriques pour le domaine plus restreint des perceptions sensorielles immédiates. À l'instar de ce dernier, qui a démontré que l'activité spécifique des organes influait sur les perceptions sensorielles, Kant a montré ce qui, dans nos perceptions, relevait des règles particulières et spécifiques de l'esprit pensant. Comme vous le voyez, les idées de Kant sont toujours vivantes et ne cessent de déployer leur richesse, même dans les domaines où l'on n'aurait peut-être pas songé à cueillir leurs fruits. J'espère ainsi vous avoir fait comprendre que l'opposition entre philosophie et sciences de la nature concernait seulement certains systèmes récents de la philosophie et non pas toute espèce de philosophie. Le lien commun susceptible d'unir toutes les sciences les unes aux autres n'est nullement rompu par les récents développements de la science de la nature. 
Car je redoute même de vous voir appliquer à mon exposé scientifique de ce jour les vers que Méphistophélès adressait aux philosophes :

Vous verrez que la moindre chose

Que vous faisiez sans y songer,

Comme de boire ou de manger,

En un, deux, trois temps se décompose ${ }^{5}$

5. NdT : Goethe, Gesammelte Werke, vol. 2: Faust, $1^{\text {re }}$ partie, Leipzig 1925, 61. Traduction de Jean Malaplate modifiée tirée de : Faust, $1^{\text {re }}$ partie, Paris : GF Flammarion, 1984, 86 . 